\title{
Transfuser ou
}

ne pas transfuser

Un choix déterminant pour

les enfants hospitalisés en

service de soins intensifs

Jacques Lacroix, Helen Trottier, Marisa Tucei

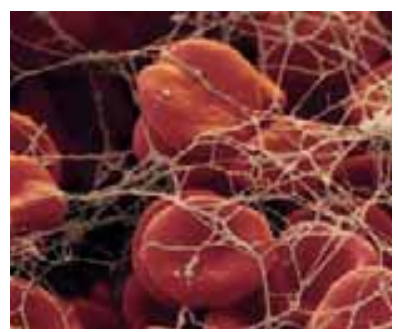

La majorité (74\%) des enfants hospitalisés en service de réanimation souffrent temporairement d'anémie (définie comme une concentration d'hémoglobine $(\mathrm{Hb})$ inférieure à $110 \mathrm{~g} / \mathrm{l})$. Près de la moitié a même besoin d'au moins une transfusion de globules rouges. Toutefois, ce type de transfusion n'est pas sans risques et aggrave même parfois l'état du patient. L'étude TRIPICU (transfusion requirements in pediatric intensive care units) [1] a démontré qu'une concentration de $70 \mathrm{~g} / \mathrm{l}$ d'Hb est à la fois suffisante, mais nécessaire, pour prévenir les effets néfastes de l'anémie et que vouloir atteindre une concentration d'Hb de $110 \mathrm{~g} / \mathrm{l}$ ne fait qu'exposer les patients à des risques inutiles.

\section{Les dangers de l'anémie}

L'Hb des globules rouges, qui capte l'oxygène dans les poumons, le transporte via la circulation sanguine et le dispense aux tissus périphériques, permet aux cellules de produire suffisamment d'ATP (la forme d'énergie utilisée par les tissus humains). Or la dépense énergétique des patients hospitalisés en réanimation est considérable à cause de la réaction inflammatoire très intense qu'ils subissent tous. Chez eux, une anémie grave peut causer un syndrome de défaillance multiviscérale (SDMV) parfois létal. Le risque mortel qu'entraîne une anémie sévère est connu: une étude faite chez des adultes en bonne santé refusant toute transfusion a montré que le risque de mortalité postopératoire augmente quand I'Hb chute en dessous de $40 \mathrm{~g} / \mathrm{l}$ [4]. Chez l'enfant malade, ce seuil est d'environ $50 \mathrm{~g} / \mathrm{l}$ [5-7].

Service des soins intensifs pédiatriques, Département de pédiatrie, Centre de recherche, Université de Montréal, CHU-Sainte-Justine 3175, Côte Sainte-Catherine, Bureau 3431, Montréal (Québec), H3T 1C3 Canada. jacques_lacroix@ ssss.gouv.qc.ca On pourrait donc s'attendre à ce que les patients dans un état encore plus précaire, comme ceux qui requièrent des soins intensifs aient besoin d'un taux d'Hb beaucoup plus élevé. Cependant, aucune donnée solide n'existait à ce sujet avant l'étude TRIPICU. En fait, la pratique variait énormément: chez l'enfant, le seuil d'Hb en dessous duquel une transfusion était préconisée variait entre 70 et $130 \mathrm{~g} / \mathrm{I}$ [8].

\section{Le revers de la médaille : les risques liés aux transfusions}

Une transfusion augmente rapidement l'Hb du receveur, puisque le taux d'Hb d'un concentré érythrocytaire (180 à $210 \mathrm{~g} / \mathrm{l})$ dépasse largement celui d'un patient anémique $(<110 \mathrm{~g} / \mathrm{l})$. On a longtemps pensé qu'il fallait transfuser tous les patients anémiques hospitalisés en soins intensifs, d'autant plus qu'on croyait la transfusion peu risquée. Malheureusement, ce n'est pas le cas. On connaît le risque de transmission d'agents infectieux, même si les mesures préventives appliquées dans les pays développés l'ont considérablement réduit (par exemple, le risque de transmettre le VIH, virus de l'immunodéficience humaine, par une transfusion était seulement de 1 pour 4,7 millions d'individus traités en 2006 au Canada [9]), mais de nombreux autres risques existent. Outre les réactions allergiques, l'hémolyse, le syndrome 


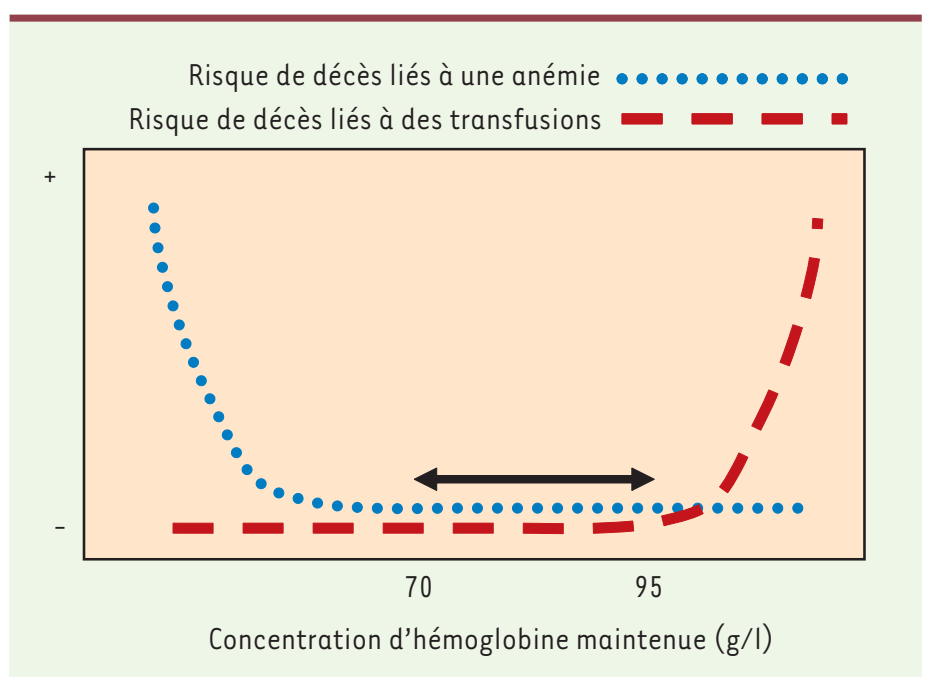

respiratoire post-transfusion, la surcharge volémique et l'hyperkaliémie, on a découvert dans les années 1990 que la transfusion de concentrés érythrocytaires pouvait exacerber une réaction inflammatoire. Or tous les patients ou presque traités en service de soins intensifs présentent un syndrome de réponse inflammatoire systémique (SRIS). Chez eux, une transfusion peut entraîner un syndrome de défaillance multiviscérale conduisant à la mort [10]. De plus, des données récentes suggèrent que les globules rouges contenus dans les concentrés érythrocytaires ne fonctionnent pas normalement et qu'ils pourraient provoquer une vasoconstriction importante chez le receveur, compromettant ainsi l'apport d'oxygène aux cellules irriguées par de petits vaisseaux sanguins [11]. La «crise énergétique cellulaire » qui en résulterait pourrait elle aussi causer un SDMV, puis éventuellement la mort (Figure 1).

\section{TRIPICU identifie le meilleur compromis pour la santé des patients}

Que faire en fin de compte? D'un côté, transfuser représente le seul moyen de corriger rapidement une anémie potentiellement nocive, de l'autre, cette opération n'est pas sans dangers. Avant notre étude, on ne connaissait pas la valeur minimale de la concentration d'Hb qui permettrait de prévenir une crise énergétique chez les patients tout en évitant le recours à des transfusions importantes.

TRIPICU est un essai clinique à répartition aléatoire multicentrique de non infériorité dont l'objectif était d'analyser l'impact de différentes concentrations d'Hb sur le sort d'enfants de moins de 15 ans traités en service de soins intensifs et stabilisés sur le plan cardiorespiratoire. Nous avons ainsi analysé l'évolution de deux groupes d'enfants chez lesquels la concentration d'Hb était maintenue à $70 \mathrm{~g} / \mathrm{l}$ (stratégie restrictive) ou $95 \mathrm{~g} / \mathrm{I}$ (stratégie libérale). Ces deux seuils (70 et 95) ont été choisis pour deux raisons: (1) ils se trouvaient dans les limites de la pratique médicale [8]; (2) nous considérions que l'écart d'Hb ( $25 \mathrm{~g} / \mathrm{l})$ serait assez grand pour observer une éventuelle différence dans le nombre et la gravité des cas de SDMV observés entre les deux groupes. Dix-neuf hôpitaux de quatre pays (États-Unis d'Amérique,
Figure 1. Comparaison des risques liés à l'anémie et aux transfusions requises pour corriger cette anémie. Le risque de mortalité liée à l'anémie ne varie presque pas au-dessus d'une certaine concentration d'hémoglobine $(\mathrm{Hb})$ (courbe en pointillés bleus), mais il augmente rapidement en-dessous de cette concentration. Pour maintenir le niveau d'Hb à une concentration donnée, il est nécessaire de répéter les transfusions. Or, plus le nombre de transfusions augmente, plus on expose le patient à des risques (ligne rouge hachurée). II est donc souhaitable de maintenir la concentration d'Hb à un seuil relativement bas offrant un compromis acceptable entre un faible risque transfusionnel l'évolution et le maintien des fonctions vitales (double flèche noire).

Canada, Royaume-Uni et Belgique) ont participé à l'étude. Les 320 patients (âgés de moins de 15 ans) chez lesquels I'Hb a été maintenue à $70 \mathrm{~g} / \mathrm{l}$ (groupe restrictif) et les 317 patients du second groupe (groupe libéral) étaient comparables au moment de la répartition aléatoire. La plupart souffraient d'insuffisance respiratoire, cardiovasculaire ou neurologique. Comme on pouvait l'anticiper, les patients du groupe restrictif ont reçu beaucoup moins de transfusions que ceux du groupe libéral (301 versus 542 transfusions au total). De plus, $54 \%$ des patients du groupe restrictif n'ont pas été transfusés alors que presque tous ceux du groupe libéral (98\%) l'ont été. Malgré ces différences, le sort des patients a été en tout point identique : la mortalité était de 14 dans les deux groupes, le nombre de cas de SDMV de 38 contre 39 (groupe restrictif versus libéral), et la durée du séjour en service de soins intensifs de $9,5 \pm 7,9$ contre $9,9 \pm 7,4$ jours.

L'étude TRIPICU a donc montré : (1) qu'il était inutile de transfuser un enfant dont l'état cardiorespiratoire est stabilisé et dont la concentration d'Hb est supérieure à $70 \mathrm{~g} / \mathrm{l}$; (2) que cette stratégie réduit de façon substantielle l'exposition de cet enfant aux risques d'une transfusion sans augmenter la morbidité et la mortalité. Au terme de notre étude, nous préconisons donc une stratégie restrictive, puisqu'elle est sans risques pour les patients et avantageuse pour les banques de sang en mal d'approvisionnement partout dans le monde. Bien que l'étude n'ait porté que sur des enfants, ses conclusions peuvent probablement être extrapolées à tous les patients traités en service de soins intensifs, à l'exception des prématurés, des vieillards et peut-être des patients souffrant d'une maladie coronarienne. $\diamond$

Improving use of red-cell transfusion

in pediatric intensive care units 


\section{CONFLIT D'INTÉRÊTS}

Les auteurs déclarent n'avoir aucun conflit d'intérêts concernant les données publiées dans cet article.

\section{RÉFÉRENCES}

1. Lacroix J, Hébert PC, Hutchison JH, et al. Transfusion strategies for patients in pediatric intensive care units. N Engl J Med 2007 ; 356 : 1609-19.

2. Bateman ST, Lacroix J, Boven K, et al. Anemia, blood loss and blood transfusion in North American children in the intensive care unit. Am J Respir Crit Care Med 2008; 178:26-33.

3. Desmet L, Lacroix J. Transfusion in pediatrics. Crit Care Clin $2004 ; 20: 299-311$.

4. Carson JL, Noveck H, Berlin JA, et al. Mortality and morbidity in patients with very low postoperative $\mathrm{Hb}$ levels who decline blood transfusion. Transfusion $2002 ; 42: 812-8$.

5. English M, Ahmed M, Ngando C, et al. Blood transfusion for severe anaemia in children in a Kenyan hospital. Lancet $2002 ; 359: 494-5$.

6. Lackritz EM, Campbell CC, Ruebush TK, et al. Effect of blood transfusion on survival among children in a Kenyan hospital. Lancet $1992 ; 340: 524-8$.
7. Lackritz EM, Hightower AW, Zucker JR, et al. Longitudinal evaluation of severely anemic children in Kenya: The effect of transfusion on mortality and hematologic recovery. AIDS $1997 ; 11$ : 1487-94.

8. Laverdière $C$, Gauvin F, Hébert PC, et al. Survey of transfusion practices in pediatric intensive care units. Pediatr Crit Care Med $2002 ; 3: 335-40$

9. Infectious diseases and immunization committee. Canadian paediatric society. Transfusion and risk of infection in Canada: update 2006. Paediatr Child Health 2006; 11 : 158-62.

10. Despond 0, Proulx F, Carcillo JA, Lacroix J. Pediatric sepsis and multiple organ dysfunction syndrome. Curr Opin Pediatr 2001; 13: 247-53.

11. Doctor A, Platt R, Sheram ML, et al. Hemoglobin conformation couples erythrocyte $\mathrm{S}$-nitrosothiol content to $\mathrm{O}_{2}$ gradients. Proc Natl Acad Sci USA $2005 ; 102: 5709-14$.

\section{TIRÉS À PART}

J. Lacroix

\section{Colloque de Recherche Biomédicale de l'ANR et Bilan des programmes 2005}

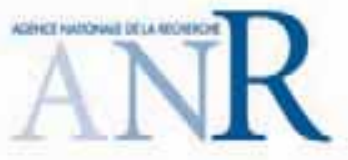

\section{6 et 27 novembre 2009}

Gité des Sciences et de l'Industrie - Centre des congrès de la Villette، Paris

Un colloque riche en événements :

Le bilan des programmes 2005 :

- Cardiologie, Obésité, Diabète

- Maladies Rares

- Microbiologie et Immunologie

- Neurosciences, Neurologie et Psychiatrie
Des sessions transversales :

- Biothérapies / cellules souches

- Canaux

- Maladies neurodégénératives

- Modèles animaux non mammifères
Des ateliers :

- Mitochondries

- Modélisation

- Santé mentale

Une exposition des 161 projets financés par l'ANR en 2005

Un forum emploi : un espace dédié afin d'organiser les rencontres et contacts entre les chercheurs 


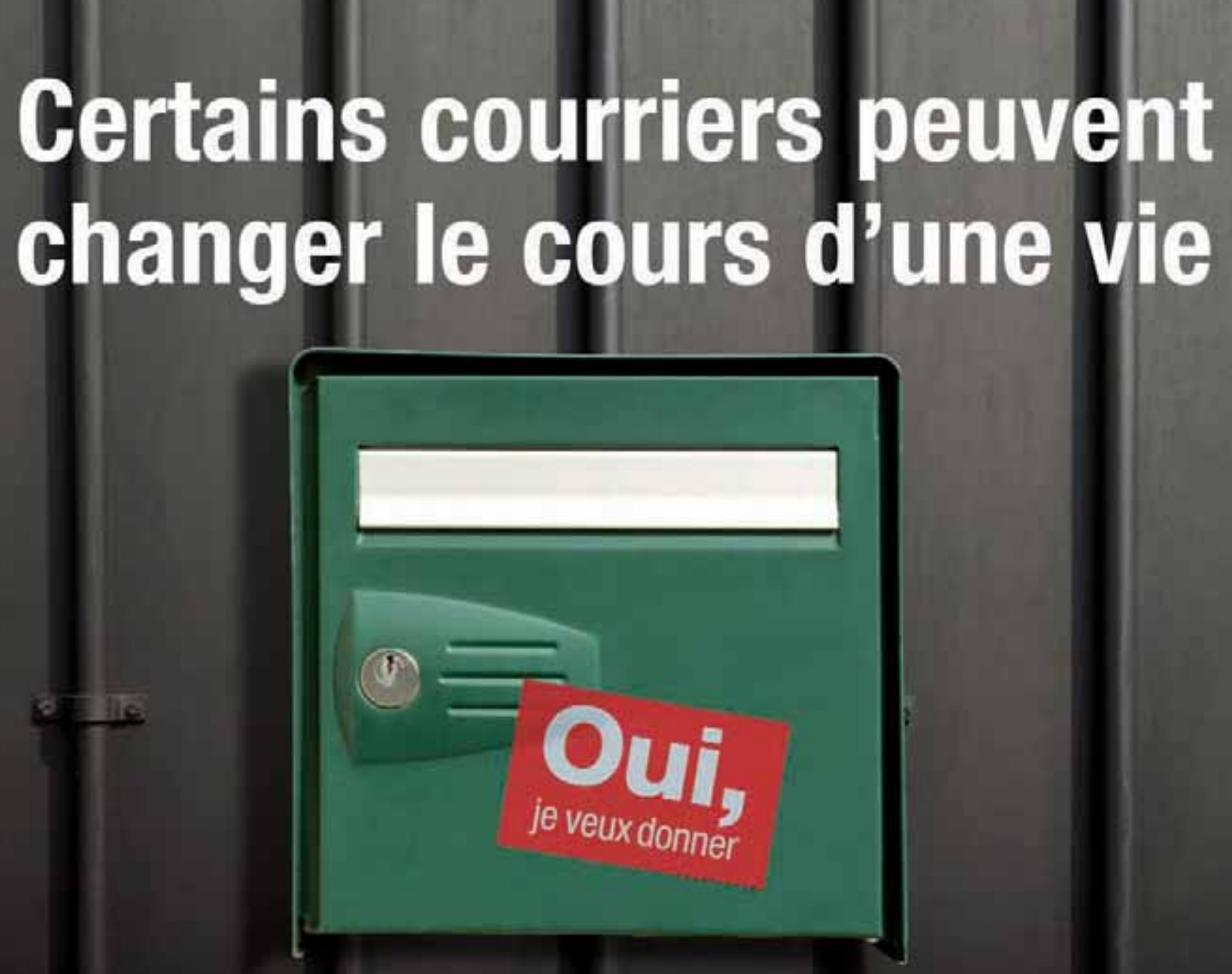

\section{Donnez-nous les moyens d'aider les plus démunis.} Répondez à notre grand appel de I'hiver en renvoyant le bulletin ci-dessous ou directement sur www-armeedusalut.fr

BULLETIN DE SOUTIEN à renvoyer avec votre don : Fondation de l'Armée du Salut - 60 rue des Frères-Flavien - 75976 Paris Cedex 20. Merci.

$\square$ U.". je participe aux actions de la Fondation de l'Armée du Salut, je fais un don dès maintenant de :

\begin{tabular}{|c|c|c|}
\hline $5 \in$ & 口 $30 €$ & D $40 € \triangle^{10 \text { repas chauds }}$ \\
\hline $0 €$ & 口 $100 €$ & autre: . \\
\hline
\end{tabular}

Par chèque bancaire ou postal à l'ordre de :

la Fondation de l'Armée du Salut

Votre don ouvre droit à une déduction d'impôt de $75 \%$ du montant de votre don (dans la limite de 510 e)

Vous pouvez exercer vos droits draccés; de rectification ou de suppression des données vous concemant dans notre fichier ( Loi L. et L. 1978). Si vous ne souhaitez pas que wotre edregse soit prététe à d'autres organismes, cocher cette case : $\square$ $\neg \mathrm{M} . \quad \neg \mathrm{Mme} \quad \square \mathrm{Mlle}$

Nom :

Prénom :

Adresse :

Code postal : $L|+|||$

Ville :

Courriel : (facultatif)

SECOURIR, ACCOMPAGNER, RECONSTRUIRE La Fondation de l'Armée du Salut

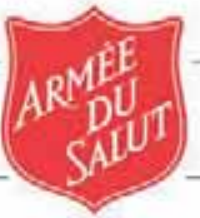

AECONNUE D UTILITE PUBLIOUE 